\title{
Cell surface antigens of neonatal monocytes are selectively impaired in basal expression, but hyperresponsive to lipopolysaccharide and zymosan
}

\section{Norikatsu Hikita, Yuki Cho, Daisuke Tachibana, Takashi Hamazaki, Masayasu Koyama, Daisuke Tokuhara}

\begin{tabular}{|c|c|}
\hline Citation & Journal of Reproductive Immunology. 136; 102614 \\
\hline Issue Date & $2019-11$ \\
\hline Type & Journal Article \\
\hline Textversion & author \\
\hline Highlights & $\begin{array}{l}\cdot \text { Neonates have limited innate immune responses to infection. } \\
\cdot \text { We compared cord blood }\left(\mathrm{CD} 14^{+} \mathrm{CD} 16^{\text {high }}\right) \text { and adult }\left(\mathrm{CD} 14^{+} \mathrm{CD} 16^{\text {intermediate }}\right) \text { monocytes. } \\
\cdot \text { Cord blood monocytes had low basal expression of MHC class II, CD } 80 \text {, and CD11b. } \\
\cdot \text { TLR4 or TLR2/6 stimulation increased the antigens more in cord than adult monocytes. } \\
\cdot \text { TLR agonists may have utility as vaccine adjuvants in infants. }\end{array}$ \\
\hline Rights & $\begin{array}{l}\text { (C) } 2019 \text { Elsevier B.V. This manuscript version is made available under the } \\
\text { CC-BY-NC-ND } 4.0 \text { License. http://creativecommons.org/licenses/by-nc-nd/4.0/. } \\
\text { This is the accepted manuscript version. Please cite only the published version. The } \\
\text { article has been published in final form at https://doi.org/10.1016/j.jri.2019.102614. }\end{array}$ \\
\hline DOI & 10.1016/j.jri.2019.102614 \\
\hline
\end{tabular}

Self-Archiving by Author(s)

Placed on: Osaka City University

HIKITA, N., CHO, Y., TACHIBANA, D., HAMAZAKI, T., KOYAMA, M., \& TOKUHARA, D. (2019).

Cell surface antigens of neonatal monocytes are selectively impaired in basal expression, but hyperresponsive to lipopolysaccharide and zymosan. Journal of Reproductive Immunology. 136, 102614. doi:10.1016/j.jri.2019.102614 
Cell surface antigens of neonatal monocytes are selectively impaired in basal expression, but hyperresponsive to lipopolysaccharide and zymosan

Norikatsu Hikita, M.D., Ph.D. ${ }^{*}$, , Yuki Cho, M.D., Ph.D. ${ }^{*}$ ), Daisuke Tachibana, M.D., Ph.D. ${ }^{2)}$, Takashi Hamazaki, M.D., Ph.D. ${ }^{1)}$, Masayasu Koyama, M.D., Ph.D. ${ }^{2)}$, Daisuke Tokuhara, M.D., Ph.D. ${ }^{1,3)}$

1) Department of Pediatrics, Osaka City University Graduate School of Medicine, Osaka, Japan

2) Department of Obstetrics and Gynecology, Osaka City University Graduate School of Medicine, Osaka, Japan

3) Department of Medicine, University of California San Diego, San Diego, CA, USA

Short title: TLR-mediated neonatal innate immunity

*Contributed equally

\section{Corresponding author:}

Daisuke Tokuhara, $\mathrm{MD}, \mathrm{PhD}$

Department of Pediatrics, Osaka City University Graduate School of Medicine,

1-4-3 Asahimachi, Abenoku, Osaka 545-8585, Japan.

E-mail: m1155519@med.osaka-cu.ac.jp; Tel: +81-6-6645-3816; Fax: +81-6-6636-8737 


\section{Abstract}

Toll-like receptors (TLRs) are important components of the innate immune system, but how neonatal TLR-mediated immune responses differ from those of adult is unknown. We aimed to clarify the TLR-mediated expression profiles of cell surface antigens related to antigen presentation in neonates. CD14-positive monocytes were isolated from human cord blood and adult peripheral blood and then stimulated with lipopolysaccharide (LPS; a TLR4 agonist) or zymosan (a TLR2/6 agonist) or left unstimulated. Expression levels of the surface antigens major histocompatibility (MHC)-class II, CD80, CD86, CD11b, CD11c, CD14, and CD16 were then evaluated by means of flow cytometry. Cord blood $\mathrm{CD} 14^{+} \mathrm{CD} 16^{\text {high }}$ monocytes $(\mathrm{CBM})$ showed significantly lower basal levels of MHC-class II, CD80, and CD11b compared with those of adult blood CD14 ${ }^{+} \mathrm{CD} 16^{\text {intermediate }}$ monocytes $(\mathrm{ABM})(\mathrm{P}<0.01, \mathrm{P}<0.001, \mathrm{P}<0.001$, respectively $)$. LPS stimulation significantly enhanced expression of MHC class II, CD80 and CD11b significantly more in $\mathrm{CBM}$ than in $\mathrm{ABM}(\mathrm{P}<0.001, \mathrm{P}<0.01, \mathrm{P}<0.01$, respectively), resulting in levels that did not differ between CBM and ABM. Zymosan stimulation also enhanced expression of MHC class II antigen, CD86, CD11b and CD11c significantly more in CBM than in ABM $(\mathrm{P}<0.001$, $\mathrm{P}<0.01, \mathrm{P}<0.001, \mathrm{P}<0.01$, respectively), resulting in levels of CD86 and CD11c that did not differ between $\mathrm{CBM}$ and $\mathrm{ABM}$. However, MHC class II, CD80 and CD11b remained significantly higher in $\mathrm{ABM}$ than in $\mathrm{CBM}(\mathrm{P}<0.05, \mathrm{P}<0.01, \mathrm{P}<0.05$, respectively). These data indicate that $\mathrm{CBM}$ and $\mathrm{ABM}$ have distinct phenotypes and responses to stimulation. 
Keywords: cord blood; innate immunity; monocyte; neonate; toll-like receptor

Abbreviations: APC, antigen presenting cell; DC, dendritic cell; mAb, monoclonal antibody;

TLR, toll-like receptor 


\section{Introduction}

Gastrointestinal and respiratory infections cause over $40 \%$ of deaths in children younger than 5 years across the world (Liu et al., 2015; Tokuhara, 2018a). The ability of neonates and infants to acquire protective immunity after infection or immunization is poor; infection leading to severe outcomes (e.g., sepsis and pneumonia) is a major cause of neonatal death ( 20\%) (Liu et al., 2015). Protective humoral immunity, represented as pathogen-specific systemic IgG and mucosal (e.g., respiratory and intestinal) IgA (Tokuhara, 2018a; Tokuhara et al., 2018b; Tokuhara et al., 2010), is induced by maturation and class-switching of B cells and mediated via T cell-dependent and/or -independent pathways. As an initial step in the both pathways, antigen-presenting cells (APCs), such as dendritic cells (DCs), recognize pathogen via toll-like receptors (TLRs); the APCs then process the pathogen-specific peptide, complex it with MHC class antigen associated with co-stimulatory molecules (CD80 and CD86), and present it to T and B cells (Guermonprez et al., 2002). Upon stimulation by pathogen, APCs also produce cytokines (e.g., IL-6), which activate B cell maturation (Tokuhara et al., 2018b). Therefore, elucidation of immune responses related to antigen presentation by APCs in neonates and infants may provide keys to understanding the mechanisms of high susceptibility to infection and facilitate development of effective vaccines in those populations.

Previous immunological studies focusing on TLR-mediated cytokine profiles of neonatal APCs have used cord blood, which is non-invasively collectable from placenta after birth and reflects neonatal immune system (Kollmann et al., 2009; Schüller et al., 2013; Levy et al., 2004; 
Nohmi et al., 2015; Yanai et al., 2016; Nguyen et al., 2010; De Wit et al., 2003). All of those studies indicated that TLR-mediated cytokine responses are not completely impaired, but rather are selectively reduced or altered, in neonates depending on gestational age, the types of APCs or TLRs, and plasma or maternal factors.

In terms of the surface antigens, a previous study disclosed that neonatal monocytes show low HLA-class II basal expression and TLR 4-and TLR 9-mediated hypoexpression of HLA-class II and CD80 compared with those in adults (Nguyen et al., 2010), whereas another study demonstrated that there were no differences in basal expression of HLA-class II, CD80, CD86, and CD40 between neonatal and adult DCs and that TLRs stimulated CD40 and CD80 hypoexpression in neonatal DCs (De Wit et al., 2003). These two studies indicate the selective impaired basal and/or TLR-stimulated expression of cell surface molecules in neonates, but further studies are necessary to confirm these issues. Therefore, here we aimed to disclose the characteristics of basal and stimulated expressions of cell surface molecules related to antigen presentation in neonatal immune cells by using purified cord blood monocytes. Our results clearly clarified the distinct phenotype of neonatal monocytes $\left(\mathrm{CD} 14^{+} \mathrm{CD} 16^{\text {high }}\right)$ compared with that of adult monocytes $\left(\mathrm{CD} 14^{+} \mathrm{CD} 16^{\text {intermediate }}\right)$. We demonstrated the low basal expression of MHCclass II, CD80, and CD11b on neonatal monocytes; and we clarified the degree to which TLR4and TLR2/6-stimulated expression of neonatal monocyte surface antigens was enhanced compared with that in adults. These results contribute to our understanding of the neonatal innate immune system and could be the foundation of developing vaccine adjuvants for children. 


\section{Materials and Methods}

\subsection{Blood samples}

Heparinized cord blood (30 to $50 \mathrm{ml}$ ) was collected during elective caesarean sections in women with healthy, full-term pregnancies $(37.8 \pm 0.4$ weeks gestational age, mean $\pm 1 \mathrm{SD}, \mathrm{n}=15)$ and without premature rupture of membranes or infection. Heparinized peripheral blood (50 to $75 \mathrm{ml}$ ) was collected from healthy adults without infection $(26.7 \pm 3.0$ years, mean $\pm \mathrm{SD}, \mathrm{n}=19$, male/female $=12 / 7)$. Mononuclear cells were separated from cord or adult peripheral blood within $2 \mathrm{~h}$ after blood collection. The study protocol conformed to the principles of the Declaration of Helsinki. The ethics committee of our institution approved the study protocol (No. 3598), and written informed consent was obtained before the procedures commenced.

\subsection{Monocyte separation}

Heparinized cord or adult blood was layered onto Lymphocyte Separation Medium (126-04871;

Wako Pure Chemical Industries Ltd., Osaka, Japan) followed by centrifugation, and the mononuclear cell layer was collected. Monocytes were isolated from mononuclear cells by positive selection using magnetic microbeads coupled to an anti-CD14 monoclonal antibody (mAb) (130-050-201; Miltenyi Biotec, Auburn, CA, USA) in accordance with the manufacturer's instructions (Nohmi et al., 2015; Yanai et al., 2016). Approximately $1 \times 10^{7}$ CD14-positive monocytes were separated from $50 \mathrm{ml}$ of cord blood. The purity of isolated monocytes $(>97 \%$ 
CD14+; >99\% cell viability) was confirmed by fluorescence-activated cell sorting (FACS; LSR

II, BD Biosciences) using APC-conjugated anti-human CD14 mAb (BD Pharmingen Catalog No:

555399) (Nohmi et al., 2015).

\subsection{TLR stimulations}

Cord blood monocytes $(\mathrm{CBM})$ and adult blood monocytes $(\mathrm{ABM})$ were separately transferred to 24-well plates $\left(10^{6}\right.$ cells/well) and incubated with the TLR4 ligand lipopolydsaccharide (LPS; $100 \mathrm{ng} / \mathrm{ml}$; derived from Escherichia coli serotype O111 B4; Sigma) or the TLR2/6 ligand zymosan (10 $\mu \mathrm{g} / \mathrm{ml}$; IMG-2212, Imgenex) for $6 \mathrm{~h}$, or incubated without treatment (control) at $37{ }^{\circ} \mathrm{C}$ in humidified air containing $5 \% \mathrm{CO}_{2}$ as previous described (Nohmi et al., 2015). The concentrations of TLR ligands used were determined by preliminary experiments.

\subsection{Flow cytometry}

All antibodies used for flow cytometry were obtained from BD Pharmingen. To characterize their phenotype, monocytes isolated from cord blood and adult peripheral blood were stained with APC-conjugated anti-human CD14 mAb (described in Monocyte separation section) and PElabeled anti-human CD16 mAb (Catalog No: 555407) prior to TLR stimulation. After TLR stimulation, to characterize the expression of cell surface antigens related to antigen presentation, cells were collected and incubated with an Ab cocktail consisting of FITC-conjugated anti-human HLA-DP, DQ, DR mAb (major histocompatibility [MHC] class II; Catalog No: 555558), PE- 
labeled anti-human CD11b mAb (Catalog No: 555388), APC-conjugated anti-human CD11c mAb (Catalog No: 559877); or PE-labeled anti-human CD80 mAb (Catalog No: 557227) and APC-conjugated anti-human CD86 mAb (Catalog No: 555660). These samples underwent FACS (LSR II, BD Biosciences) and the mean fluorescent intensity (MFI) scores or the percent positive cells were calculated by Flowjo software (version 10.4.2). TLR-mediated expression of cell surface antigens with and without TLR stimulation are reported as MFI. In addition, enhancement ratios were calculated as [MFI after TLR stimulation] divided by [MFI without TLR stimulation].

\subsection{Cytokine assay}

The concentrations of human IL-8, IL-1 $\beta$, IL-6, and tumor necrosis factor (TNF)- $\alpha$ in the cellculture supernatants and serum from cord and adult blood were measured by using a human inflammatory cytokine cytometric bead assay kit (catalog no. 551811, BD Pharmingen, San Jose, CA, USA) in accordance with the manufacturer's instructions (Nohmi et al., 2015; Yanai et al., 2016). Briefly, $50 \mu \mathrm{l}$ of supernatant was mixed with $50 \mu \mathrm{l}$ of mixed capture beads. After $3 \mathrm{~h}$ of incubation at room temperature, the samples were washed, suspended in phosphate-buffered saline, and then analyzed by flow cytometry (FACS LSR II, Becton Dickinson, Franklin Lakes, NJ, USA) and the CBA Analysis Software (BD Biosciences). 


\subsection{Statistics}

The differences in expression levels of surface molecules between cord and adult blood monocytes were analyzed by using the Mann-Whitney $U$ test. Values of $P<0.05$ were considered statistically significant. Statistical significance is expressed in the Figures and Tables through the use of asterisks $(* \mathrm{P}<0.05, * * \mathrm{P}<0.01, * * * \mathrm{P}<0.001)$.

\section{Results}

\subsection{Characteristics of neonates and cord blood}

All newborns were healthy, with an Apgar score (mean \pm 1 SD) of $8.7 \pm 0.5$ at $5 \mathrm{~min}$. None of the newborns had macrosomia, cardiac anomaly, respiratory distress syndrome, or infectious diseases. Their cord blood $\operatorname{IgM}($ mean $\pm 1 \mathrm{SD}$ ) was $8.7 \pm 2.8 \mathrm{mg} / \mathrm{dL}$ (range, 5 to 15 ; normal upper limit, 20), indicating a lack of intrauterine or peripheral infection. The serum from the cord blood showed no excessive elevation of inflammatory cytokines (IL8, TNF-alpha, IL-1 beta, IL-6), and the cytokine concentrations were not statistically different from those in adult serum (Supplementary Table 1). These results indicate that CBM used in this study were not activated by occult infection or premature rupture of membrane.

\subsection{MHC-class II, CD80, and CD11b basal expression levels were low in CBM}

The basal expression levels of MHC class II antigen, CD80, and CD11b were significantly lower in $\mathrm{CBM}$ than in $\mathrm{ABM}(\mathrm{P}<0.01, \mathrm{P}<0.001, \mathrm{P}<0.001$, respectively), whereas there was no 
significant difference in basal levels of CD86 and CD11c between the two types of monocytes (Fig. 1 and summarized in Table 1).

Table 1. Summary of the expression of cell surface antigens.

\begin{tabular}{cccccc}
\hline & \multicolumn{5}{c}{ MFI } \\
\cline { 2 - 6 } & HLA & CD80 & CD86 & CD11b & CD11c \\
\hline Non-treated & CBM $<$ ABM & CBM $<$ ABM & NS & CBM $<$ ABM & NS \\
LPS-stimulated & $\mathrm{NS}$ & $\mathrm{NS}$ & $\mathrm{NS}$ & $\mathrm{CBM}<\mathrm{ABM}$ & $\mathrm{NS}$ \\
Zymosan-stimulated & $\mathrm{CBM}<\mathrm{ABM}$ & $\mathrm{CBM}<\mathrm{ABM}$ & $\mathrm{NS}$ & $\mathrm{CBM}<\mathrm{ABM}$ & $\mathrm{NS}$ \\
LPS /non-treated & $\mathrm{CBM}>\mathrm{ABM}$ & $\mathrm{CBM}>\mathrm{ABM}$ & $\mathrm{NS}$ & $\mathrm{CBM}>\mathrm{ABM}$ & $\mathrm{NS}$ \\
Zymosan /non-treated & $\mathrm{CBM}>\mathrm{ABM}$ & $\mathrm{NS}$ & $\mathrm{CBM}>\mathrm{ABM}$ & $\mathrm{CBM}>\mathrm{ABM}$ & $\mathrm{CBM}>$ \\
& & & & & $\mathrm{ABM}$
\end{tabular}

MFI, mean fluorescence intensity; ABM, adult blood monocytes; CBM, cord blood monocytes; LPS, lipopolysaccharide; NS, not significant

3.3. LPS or zymosan stimulation selectively enhanced the surface molecule expression in CBM and $A B M$

Compared with the non-treated samples, LPS stimulation significantly enhanced the expression levels of the surface antigens MHC class II antigen, CD80, CD11b, and CD11c $(\mathrm{P}<0.05, \mathrm{P}<$ 0.001, $\mathrm{P}<0.01, \mathrm{P}<0.01$, respectively), but not CD86 ( $\mathrm{P}>0.05)$, in CBM, whereas it significantly 
enhanced the expression levels of CD80, CD11b, and CD11c $(\mathrm{P}<0.001, \mathrm{P}<0.05, \mathrm{P}<0.01$, respectively), but not MHC class II antigen or CD86 (P > 0.05), in ABM (Fig. 1a).

To determine how much LPS enhances the surface molecule expression compared with the basal levels, we calculated enhancement ratios (Table 1 and Supplementary Fig. 1). CBM showed significantly higher enhancement ratios than $\mathrm{ABMs}$ for expressions of MHC class II antigen, CD80 and CD11b (P < 0.001, $\mathrm{P}<0.01, \mathrm{P}<0.01$, respectively), but not for CD86 and CD11c (P $>0.05$ ) (Supplementary Fig. 1). These results demonstrated that LPS enhanced the expression of antigen-presentation-related surface antigens more in CBM than in ABM.

In contrast, the expression levels after LPS stimulation were not statistically different between $\mathrm{CBM}$ and $\mathrm{ABM}$ for MHC class II, CD80, CD86, and CD11c and were higher in ABM than in CBM for CD11b $(\mathrm{P}<0.01$ ) (Fig. 1). Thus, despite the strong enhancement ratios in CBM, the low basal expression of these surface antigens meant that their expression in CBM after stimulation was not remarkably high.

Zymosan stimulation provided results similar to those of LPS. Zymosan stimulation significantly enhanced the expression levels of MHC class II, CD80 and CD11c in CBM (P < 0.05, $\mathrm{P}<0.001, \mathrm{P}<0.01$, respectively) but not CD86 or CD11b ( $\mathrm{P}>0.05)$ (Fig. 1b). In ABM, zymosan significantly enhanced the expression levels of CD80 and CD11c $(\mathrm{P}<0.001$ and $\mathrm{P}<$ 0.05, respectively), but not MHC class II antigen, CD86 or CD11c (P > 0.05) (Fig. 1b).

In response to zymosan stimulation, $\mathrm{CBM}$ showed significantly higher enhancement ratios than ABM for expressions of MHC class II antigen, CD86, CD11b and CD11c $(\mathrm{P}<0.001, \mathrm{P}<$ 
0.01, $\mathrm{P}<0.001, \mathrm{P}<0.01$, respectively), but not for CD80 (P > 0.05) (Supplementary Fig. 1 and

Table 1). These results demonstrated that zymosan enhanced antigen-presentation-related surface antigens more in $\mathrm{CBM}$ than in $\mathrm{ABM}$.

In contrast to the enhancement ratios, the expression levels after zymosan stimulation were not statistically not different between CBM and ABM for CD86 and CD11c, and were higher in ABM than CBM for MHC class II, CD80, and CD11c, and were higher in ABM than CBM for MHC class II, CD80, and CD11b ( $\mathrm{P}<0.05, \mathrm{P}<0.01, \mathrm{P}<0.05$, respectively) (Fig. 1b). Together with the results of the LPS stimulation, these results suggest that, despite the strong enhancement ratios in $\mathrm{CBM}$, the low basal expression of these surface antigens in $\mathrm{CBM}$ meant that their expression after stimulation was not remarkably high.

\subsection{CD16 expression is different between CBM and ABM}

Because the basal levels and degrees of stimulation of various surface antigens related to antigen presentation were different between CBM and ABM, we used FACS to examine whether the phenotype (CD14 and CD16) differed between these two types of monocytes. CBM showed a $\mathrm{CD} 14^{\text {high }} \mathrm{CD} 16^{\text {high }}$ phenotype, whereas $\mathrm{ABM}$ consisted of $\mathrm{CD} 14^{\text {high }} \mathrm{CD} 16^{\text {intermediate }}$ cells before TLR stimulation (Fig. 2). After TLR stimulations by LSP or zymosan, CD16 expression on the CD14+ CBM reduced to a level (CD16 intermediate) statistically similar to that on CD14+ ABM. 


\subsection{Cytokine profiles after TLR stimulation}

Inflammatory cytokines (IL-8, IL-6, IL-1-beta and TNF-alpha) were remarkably elevated after $6 \mathrm{~h}$ of LPS or zymosan stimulation in both CBM and ABM (Fig. 3.). There were no significant differences in cytokine concentration between CBM and ABM, except that the concentration of TNF-alpha was higher in ABM than in CBM after zymosan stimulation $(\mathrm{P}<0.05)$. These results suggest that the differences in innate immunity between neonates and adults is due to the expression of surface antigens rather than cytokine profiles after $6 \mathrm{~h}$ of TLR-stimulation.

\section{Discussion}

Innate immune responses mediated by APCs are an important initial step in inducing protective immunity against pathogens, but the TLR-mediated expression levels of cell surface antigens on neonatal APCs are not fully understood. Here, by using cord blood, we demonstrated that neonatal monocytes have a distinct phenotype and response compared with adult monocytes: basal expression levels of cell surface antigens related to antigen presentation were selectively prone to be low but hyperresponsive to TLR stimulation in neonatal CD $14^{+} \mathrm{CD} 16^{\text {high }}$ monocytes compared with adult $\mathrm{CD} 14^{+} \mathrm{CD} 16^{\text {intermediate }}$ monocytes.

We observed low basal expression of the surface antigens MHC class II antigen, CD80, and CD11b, but not CD11c and CD86, on neonatal monocytes. Previously, Nguyen et al. reported that basal expression of MHC class II antigen, but not CD80, is low on neonatal monocytes and DCs (Nguyen et al., 2010), whereas De Wit et al. reported comparable basal expression of MHC 
class II antigen, CD80, and CD86 on neonatal DCs compared with adults (De Wit et al., 2003).

Taken together these findings suggest that not all neonatal surface antigens related to the antigen presentation are impaired, but rather some antigens are selectively low, especially MHC class II antigen. Comparison of basal CD11b expression on monocytes or DCs between neonates and adults has not been described previously. Our observation of low expression of CD11b in neonatal monocytes indicates the immaturity of these monocytes; such immaturity may also explain the observed selective impairment of MHC class II antigen and CD80.

Neonates are susceptible to infections and weak to gain the vaccine efficacy. Those issues let us hypothesize that low basal expressions of surface antigens (MHC class II, etc) are involved in the underlying mechanisms. MHC class II antigen and CD80 are key molecules to provide a peptide of pathogen or vaccine antigen to $\mathrm{B}$ and $\mathrm{T}$ cells followed by the initiation of acquired immunity. Previous study demonstrated that vaccine-mediated acquisiton of protective immunity without adjuvant is impaired in neonates compared to those of infants (Lieberman JM et al., 1995; Kurikka S et al., 1995) and multiple booster shots are needed to sustain immune responses and maintain immunological memory in this population (PrabhuDas $M$ et al., 2011). Although the transfer of maternal antibodies is believed to influence on the induction of vaccine-induced specific immunity, our results implicate that selective impairment in basal expression of MHCclass II antigen and CD80 will be also involved in the poor recognition of vaccine antigen by antigen-presenting cells in neonates followed by the insufficient induction of specific immunity. 
In terms of the clinical significance of low MHC class II antigen expression, a previous study demonstrated the close relationship between low expression of MHC class II antigen on monocytes and the frequency of severe respiratory syncytial virus infection (Ahout et al., 2016). Reduced MHC class II antigen expression is also a characteristic of MHC-class II deficiency (bare lymphocyte syndrome), which is associated with increased infectious susceptibility and intractable diarrhea (Kuo et al., 2013). Because the primary function of MHC class II antigen is to present peptide antigen to T cells, low MHC class II antigen expression would be expected to result in insufficient induction of protective immunity. Although the low MHC class II antigen expression observed here in the neonatal period is possibly a transient phenomenon that will be upregulated with maturation to levels comparable to that in adults during the first year of life (De Wit et al., 2003), we propose that the low MHC class II antigen expression during the neonatal period likely explains the high susceptibility to infection and the high risk of developing severe outcome in neonates and infants (Liu et al., 2015), and could be the underlying mechanism of the weak induction of protective immunity by vaccination and the need for repeated vaccinations in infants (Tokuhara, 2018a; Mendelman et al., 2001; Hill et al., 2006).

On the other hand, immune hyperresponsiveness by TLR stimulation in CBM let us consider both beneficial and harmful effects of TLR in neonates. Beneficial aspect is the application of TLR as a vaccine adjuvant. Current strategies for developing safe vaccines tend to use an isolated protein of the pathogen (e.g., virus-like particle) that is less antigenic compared with the whole pathogen vaccine (Tokuhara, 2018a); such vaccines need an effective adjuvant to 
provoke innate immune response and enable APCs to recognize vaccine antigen in neonates and infants. Our finding that the degree of responsiveness to TLR4 and TLR2/6 agonists, LPS and zymosan, was significantly higher in neonates than adults, suggests that TLR agonists could be used as a vaccine adjuvant to complement the weak antigen presentation capacity of neonatal and infantile APCs, providing effective antigen presentation and inducing acquired immunity. On the contrary, TLR4 and TLR2/6 agonists didn't enhance MHC-class II expression in adult monocytes, thus a strategy of using those TLR agonists as vaccine adjuvants may not be effective in adult population.

In terms of the harmful aspect, TLR-mediated hyperresponsiveness in surface antigens may be interacted with exacerbation of inflammation in immune diseases or multiple organ injury during sepsis. A previous study described that sepsis was associated with an increase in expression of CD80 not but CD86 (Nolan A, 2008). Another study disclosed that CD11b density increase before the arousal of the sepsis thus can be used for the earlier diagnosis of late-onset infection in extremely low birth-weight infants (Turunen R, 2005). Gram-negative bacterial infection and fungal infection are important infection high mortaliry rate in neonatal period. LPS and zymosan, cell wall component for gram-negative bacteria and fungus respectively, significantly enhanced expressions of CD11b and/or CD80 in CBM compared with ABM. Those phenomen may reflect that neonates prone to develop sepsis.

Monocytes are classically defined as CD14+ cells, but CD16 also characterizes the phenotype of monocytes (Gordon and Taylor, 2005; Ziegler-Heitbrock, 2007). Here, we 
demonstrated that CD16 expression on neonatal CD14+ monocytes was significantly higher than on adult $\mathrm{CD} 14+$ monocytes. Adult monocytes with the phenotype $\mathrm{CD} 14^{+} \mathrm{CD} 16^{\text {low to intermediate }}$ are considered to be classical monocytes (Gordon and Taylor, 2005; Ziegler-Heitbrock, 2007), but the neonatal monocytes in the current study showed the distinct phenotype of CD $14^{+} \mathrm{CD} 16^{\text {high }}$. In adults, another type of monocyte $\left(\mathrm{CD} 14^{\text {low }} \mathrm{CD} 16^{\text {high }}\right)$, which was not found in our study, is known to show higher antigen-presentation capacity (e.g., high MHC class II expression) compared with classical monocytes. Despite the higher CD16 expression on neonatal monocytes compared with adult monocytes, basal MHC-class II expression was significantly lower in neonatal monocytes $\left(\mathrm{CD} 14^{+} \mathrm{CD} 16^{\text {high }}\right)$, as mentioned above. Furthermore, TLR stimulation resulted in the reduction of $\mathrm{CD} 16$ expression in $\mathrm{CBM}$ to the same level (low to intermediate) as well as ABM, therefore initial phyenotype $\left(\mathrm{CD} 14^{+} \mathrm{CD} 16^{\text {high }}\right)$ of neonatal monocytes may reflect the immature status compared to $\mathrm{CD} 14^{+} \mathrm{CD} 16^{\text {low to intermediate }}$ adult monocytes.

The current study has some limitations in terms of evaluated immune cells and TLRs. There are ten types of TLRs in humans (Kawai and Akira, 2010), but because of limited resources, we chose to focus on TLR4 and TLR2/6, which recognize E. coli and group B streptococcus (Henneke et al., 2008; Kaper et al., 2004), respectively, important pathogens causing meningitis in neonates and infants (Stoll et al., 2011). In addition, our study evaluated monocytes but not DCs because of the abundance of monocytes and extremely low numbers of DCs in cord and adult blood. Elucidation of innate immune responses in neonatal DCs and via other TLRs will be achieved in future studies. 
In conclusion, we demonstrated that neonatal CD14+ monocytes have a distinct phenotype and response compared with those of adults. Cell surface antigens of neonatal CD $14^{+} \mathrm{CD} 16^{\text {high }}$ monocytes are selectively impaired in basal expression (MHC-class II, CD80 and CD11b) but are hyperresponsive to LPS and zymosan compared with adult CD $14^{+} \mathrm{CD} 16^{\text {intermediate }}$ monocytes. These results improve our understanding of neonatal and infantile immature innate immunity and raise the possibility of the application of TLR agonists for complementing the induction of protective immunity as a vaccine adjuvant in children.

Disclosure: None of the authors has any conflict of interest related to this manuscript.

\section{Acknowledgements}

This work was supported by grants from the Japan Society for the Promotion of Science (Grantin-Aid for Scientific Research (C)16K10039 [to D.Tokuhara] and Fund for the Promotion of Joint International Research 17KK0196 [to D.Tokuhara]), from Takano Life Science Research Foundation (to D.Tokuhara) and from the Danone Institute of Japan Foundation (to D.Tokuhara). 


\section{References}

Ahout, I.M., Jans, J., Haroutiounian, L., Simonetti, E.R., van der Gaast-de Jongh, C., Diavatopoulos, D.A., et al., 2016. Reduced Expression of HLA-DR on Monocytes During Severe Respiratory Syncytial Virus Infections. Pediatr. Infect. Dis. J. 35(3), e89-96.

De Wit, D., Tonon, S., Olislagers, V., Goriely, S., Boutriaux, M., Goldman, M., Willems, F., 2003. Impaired responses to toll-like receptor 4 and toll-like receptor 3 ligands in human cord blood. J. Autoimmun. 21, 277-81.

Gordon, S., Taylor, P.R., 2005. Monocyte and macrophage heterogeneity. Nat. Rev. Immunol. 5, 953-964.

Guermonprez, P., Valladeau, J., Zitvogel, L., Théry, C., Amigorena, S., 2002. Antigen presentation and T cell stimulation by dendritic cells. Annu. Rev. Immunol. 20, 621-667.

Henneke, P., Dramsi, S., Mancuso, G., Chraibi, K., Pellegrini, E., Theilacker, C., et al., 2008. Lipoproteins are critical TLR2 activating toxins in group B streptococcal sepsis. J. Immunol. 180, 6149-58.

Hill, D.R., Ford, L., Lalloo, D.G., 2006. Oral cholera vaccines: use in clinical practice. Lancet. Infect. Dis. 6, 361-373.

Kaper, J.B., Nataro, J.P., Mobley, H.L., 2004. Pathogenic Escherichia coli. Nat. Rev. Microbiol. 2, 123-140.

Kawai, T., Akira, S., 2010. The role of pattern-recognition receptors in innate immunity: update on Toll-like receptors. Nat. Immunol. 11, 373-384. 
Kollmann, T.R., Crabtree, J., Rein-Weston, A., Blimkie, D., Thommai, F., Wang, X.Y., et al., 2009. Neonatal innate TLR-mediated responses are distinct from those of adults. J. Immunol.

$183,7150-7160$.

Kuo, C.Y., Chase, J., Garcia Lloret, M., Stiehm, E.R., Moore, T., Aguilera, M.J., et al., 2013. Newborn screening for severe combined immunodeficiency does not identify bare lymphocyte syndrome. J. Allergy. Clin. Immunol. 131, 1693-1695.

Levy, O., Zarember, K.A., Roy, R.M., Cywes, C., Godowski, P.J., Wessels, M.R., 2004. Selective impairment of TLR-mediated innate immunity in human newborns: neonatal blood plasma reduces monocyte TNF-alpha induction by bacterial lipopeptides, lipopolysaccharide, and imiquimod, but preserves the response to R-848. J. Immunol. 173, 4627-4634.

Liu, L., Oza, S., Hogan, D., Perin, J., Rudan, I., Lawn, J.E., et al., 2015. Global, regional, and national causes of child mortality in $2000-13$, with projections to inform post-2015 priorities: an updated systematic analysis. Lancet. 385, 430-440.

Mendelman, P.M., Cordova, J., Cho, I., 2001. Safety, efficacy and effectiveness of the influenza virus vaccine, trivalent, types $\mathrm{A}$ and $\mathrm{B}$, live, cold-adapted (CAIV-T) in healthy children and healthy adults. Vaccine. 19, 2221-2226.

Nguyen, M., Leuridan, E., Zhang, T., De Wit, D., Willems, F., Van Damme, P., et al., 2010. Acquisition of adult-like TLR4 and TLR9 responses during the first year of life. PLoS. One. 5, e10407. 
Nohmi, K., Tokuhara, D., Tachibana, D., Saito, M., Sakashita, Y., Nakano, A., et al., 2015. Zymosan induces immune responses comparable with those of adults in monocytes, dendritic cells, and monocyte-derived dendritic cells from cord blood. J. Pediatr. 167, 155-162.

Schüller, S.S., Sadeghi, K., Wisgrill, L., Dangl, A., Diesner, S.C., Prusa, A.R., et al., 2013. Preterm neonates display altered plasmacytoid dendritic cell function and morphology. J. Leukoc. Biol. 93, 781-788.

Stoll, B.J., Hansen, N.I., Sánchez, P.J., Faix, R.G., Poindexter, B.B., Van Meurs, K.P., et al., 2011. Eunice Kennedy Shriver National Institute of Child Health and Human Development Neonatal Research Network. Early onset neonatal sepsis: the burden of group B Streptococcal and E. coli disease continues. Pediatrics. 127, 817-826.

Tokuhara, D., Yuki, Y., Nochi, T., Kodama, T., Mejima, M., Kurokawa, S., et al., 2010. Secretory IgA-mediated protection against $\mathrm{V}$. cholerae and heat-labile enterotoxin-producing enterotoxigenic Escherichia coli by rice-based vaccine. Proc. Natl. Acad. Sci. U S A. 107, 87948799.

Tokuhara, D., 2018a. Challenges in developing mucosal vaccines and antibodies against infectious diarrhea in children. Pediatr. Int. 60, 214-223.

Tokuhara, D., Kurashima, Y., Kamioka, M., Nakayama, T., Ernst, P., Kiyono, H., 2018b. A comprehensive understanding of the gut mucosal immune system in allergic inflammation. Allergol. Int. 68, 17-25. 
Yanai, S., Tokuhara, D., Tachibana, D., Saito, M., Sakashita, Y., Shintaku, H., et al., 2016. Diabetic pregnancy activates the innate immune response through TLR5 or TLR1/2 on neonatal monocyte. J. Reprod. Immunol. 117, 17-23.

Ziegler-Heitbrock, L., 2007. The CD14+ CD16+ blood monocytes: their role in infection and inflammation. J. Leukoc. Biol. 81, 584-592.

Lieberman, J.M., Greenberg, D.P., Wong, V.K., Partridge, S., Chang, S.J., Chiu, C.Y., et al., 1995. Effect of neonatal immunization with diphtheria and tetanus toxoids on antibody responses to Haemophilus influenzae type b conjugate vaccines. J. Pediatr. 126, 198-205.

Kurikka, S., Käyhty, H., Peltola, H., Saarinen, L., Eskola, J., Mäkelä, P.H. 1995. Neonatal immunization: response to Haemophilus influenzae type b-tetanus toxoid conjugate vaccine. Pediatrics. 95, 815-22.

PrabhuDas, M., Adkins, B., Gans, H., King, C., Levy, O., Ramilo, O., et al., 2011. Challenges in infant immunity: implications for responses to infection and vaccines. Nat. Immunol. 12, 189-94. Nolan, A., Weiden, M., Kelly, A., Hoshino, Y., Hoshino, S., et al., 2008. CD40 and CD80/86 act synergistically to regulate inflammation and mortality in polymicrobial sepsis. Am. J. Respir. Crit. Care. Med. 177, 301-308.

Wakefield, C.H., Carey, P.D., Foulds, S., Monson, J.R., Guillou, P.J. 1993. Changes in major histocompatibility complex class II expression in monocytes and T cells of patients developing infection after surgery. Br. J. Surg. 8, 205-9. 
Turunen, R., Andersson, S., Nupponen, I., Kautiainen, H., Siitonen, S., Repo, H. 2005. Increased CD11b-density on circulating phagocytes as an early sign of late-onset sepsis in extremely lowbirth-weight infants. Pediatr Res. 57, 270-5. 
Figure legends

Fig. 1. Cell surface antigen profiles after LPS (TLR4 agonist) or zymosan (TLR2/6 agonist) stimulation.

a. Comparison of expressions of cell surface antigens (MHC class II, CD80, CD86, CD11b and CD11c) between cord blood monocytes and adult blood monocytes under 6 hours of LPS

(TLR4 agonist) stimulation or non-treatment condition.

b. Comparison of expressions of cell surface antigens (MHC class II, CD80, CD86, CD11b and CD11c) between cord blood monocytes and adult blood monocytes under 6 hours of zymosan (TLR2/6 agonist) stimulation or non-treatment condition.

Data are means \pm SD of mean fluorescent intensity (MFI; $\mathrm{n}=15$ and 19 for cord blood and adult blood, respectively). ${ }^{*} P<0.05,{ }^{*} * P<0.01,{ }^{* * *} P<0.001, N S$, not significant, $P$ values are from the Mann-Whitney $U$ test. LPS, lipopolysaccharide ; C, cord blood monocytes ; A, adult blood monocytes

Figure 1.
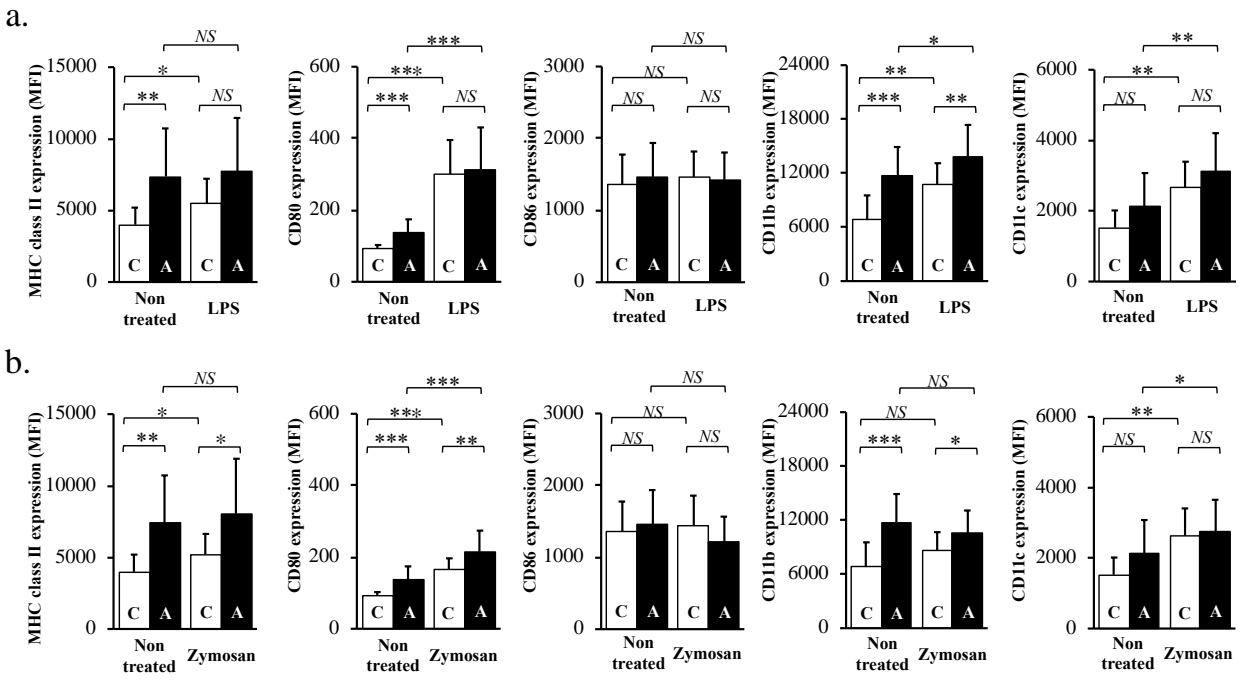
Fig. 2. CD14 and CD16 profiles in CBM and ABM.

a. CD14 and CD16 profiles in cord blood monocytes (CBM) and adult blood monocytes (ABM) isolated by using anti-CD14 magnetic beads. Percentage numbers in the right upper quadrant represent the CD14- and CD16-double positive fluorescent densities. Percentage numbers in the left upper quadrant represent the CD14- positive and CD16-negative fluorescent densities. b. Mean fluorescent intensity (MFI) of CD16 was compared between cord and adult blood CD14+ monocytes. Data are means $\pm \mathrm{SD}(\mathrm{n}=9)$. The $P$ value is from the Mann-Whitney $U$ test.

Figure 2.

a.

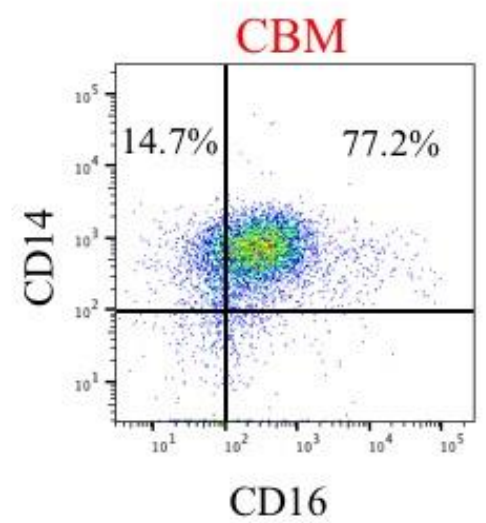

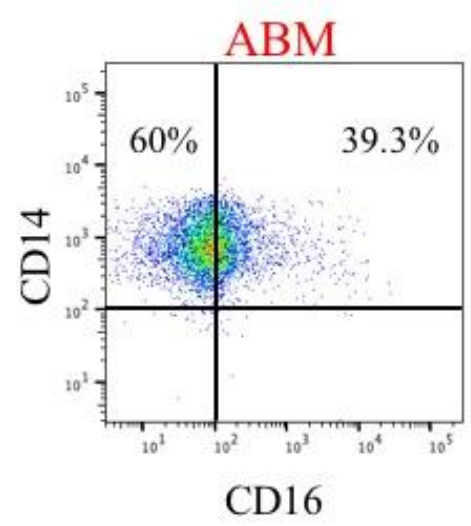

b.

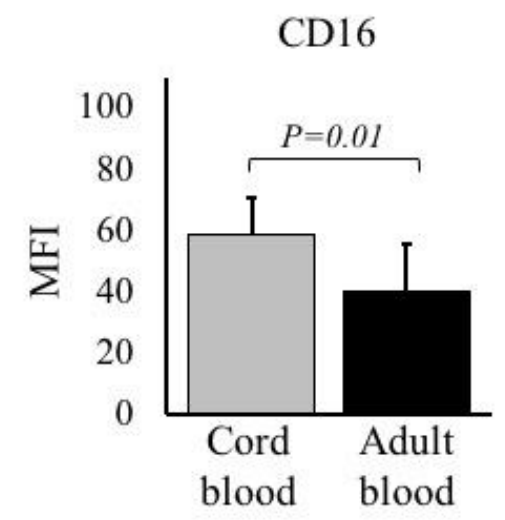


Fig. 3. Cytokine profiles after LPS (TLR4 agonist) or zymosan (TLR2/6 agonist) stimulation.

Cytokine concentrations (IL-8, IL-1 $\beta$, IL-6 and TNF- $\alpha$ ) in cell culture supernatant were determined after 6 hours of LPS (a) or zymosan (b) stimulation. Data were expressed as means \pm SD. ${ }^{*} P<0.05, N S$, not significant, $P$ values are from the Mann-Whitney $U$ test. IL, interleukin ; TNF- $\alpha$, tumor necrosis factor- $\alpha$; LPS, lipopolysaccharide ; C, cord blood monocytes ; A, adult blood monocytes

Figure 3.

a.
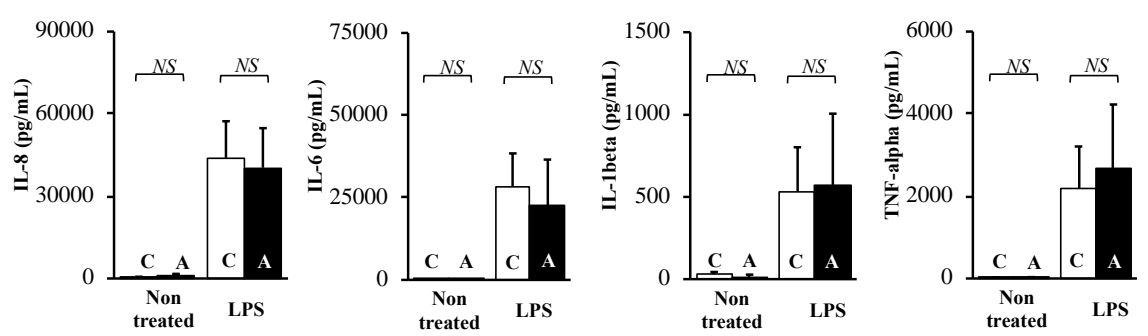

b.
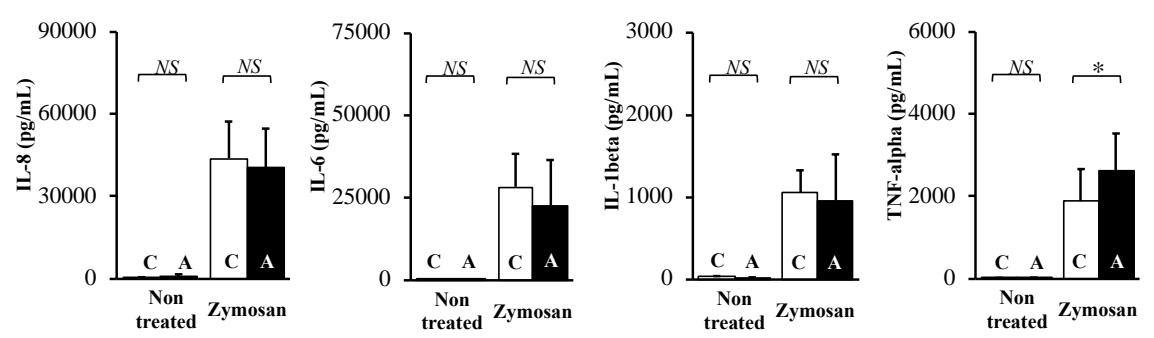